\title{
PRATIQUES ET SIGNIFICATIONS DE L'ACTIVITÉ CULINAIRE : UNE APPROCHE COMPARATIVE ENTRE DES FEMMES FRANÇAISES ET BRÉSILIENNES
}

\author{
Maria Clara de Moraes Prata Gaspar ${ }^{1}$ \\ Ursula Verthein $^{2}$
}

\section{Introduction}

La socio-anthropologie de l'alimentation pense l'acte alimentaire dans sa dimension bio-psycho-socio-culturelle (Morin, 1973) et comme un « fait social total» (Mauss, 2009 [1934]), qui participe à la construction des identités sociales (Mead, 1971 ; Poulain, 2002a ; Contreras et Gracia-Arnaiz, 2005). Dans cette perspective, la dimension culinaire représente un aspect fondamental des modèles alimentaires ${ }^{3}$ et constitue un élément du sentiment collectif d'appartenance (Fischler, 1990 ; Poulain, 2002a). Différents auteurs des champs de la sociologie et de l'anthropologie ont cherché à conceptualiser les termes « culinaire » et « cuisine », insérant ces catégories dans les discussions des sciences humaines et sociales (Jomori et al., 2018). Lévi-Strauss (1964) considère que la cuisine est une activité intermédiaire entre la nature et la culture; le culinaire serait associé à un ensemble de procédures qui transforme la nature (le cru) en culture (le cuit), c'est-à-dire la façon dont des produits crus deviennent cuisinés au travers d'un processus de transformation culturelle. Fischler (1990) postule que la « cuisine » ne peut pas seulement être définie comme un ensemble d'ingrédients et de techniques utilisés dans la préparation alimentaire, mais aussi comme un ensemble de représentations, croyances et pratiques qui lui est associé et qui fait l'objet d'un partage entre les membres d'un groupe donné. Mennel et al. (1993: 20) parlent de « culture

\footnotetext{
${ }^{1}$ Universitat de Barcelona, Espanha. Email: ma.prata@gmail.com ORCID id: https://orcid.org/0000-0002-1517-4981

${ }^{2}$ Universitat de Barcelona, Espanha. Email : ursulaverthein@gmail.com ORCID id: https://orcid.org/0000-0002-1304-9642

${ }^{3}$ Nous utilisons la notion de «modèle alimentaire » dans le sens proposé par Poulain (2002b : 25) : « des ensembles sociotechniques et symboliques qui articulent un groupe humain à son milieu, fondent son identité et assurent la mise en place de processus de différenciation interne. Ils sont un corps de connaissances technologiques, accumulées de génération en génération, permettant de sélectionner les ressources dans un espace naturel, de les préparer pour en faire des aliments, puis des plats, et de les consommer. Mais ils sont en même temps des systèmes de codes symboliques qui mettent en scène les valeurs d'un groupe humain participant à la construction des identités culturelles et aux processus de personnalisation ».
} 
culinaire » comme « a shorthand term for the ensemble of attitudes and tastes people bring to cooking and eating ». Maciel (2004), en se basant sur la distinction entre « aliment » et « nourriture » proposée par DaMatta (1986), suggère que le « culinaire » renvoie aux manières de «faire » l'aliment, de le transformer en nourriture, devenant ainsi plus qu'une simple substance comestible. Selon Maciel, ces formes de « faire » la nourriture seraient associées aux significations attribuées aux aliments et à l'acte alimentaire, et correspondraient à des styles de vie particuliers.

Les termes «culinaire » et « cuisine » sont donc mobilisés à travers différentes significations. Pourtant, ces définitions se rejoignent sur l'accent mis au-delà des dimensions matérielles, adossant des dimensions sociales, culturelles et symboliques liées au manger et au processus de préparation culinaire. Dans cette étude, c'est à partir de cette perspective que nous considérons l'activité culinaire ${ }^{4}$ comme une activité qui comprend l'ensemble des étapes de préparation alimentaire (l'approvisionnement, la préparation et la consommation), intégrée dans un système symbolique (Fischler, 1990) et expérimentée, représentée et signifiée différemment selon les circonstances temporelles et sociales (Poulain, 2002b; Kaufmann, 2005; Daniels et al., 2012 ; Adamiec, 2016), ainsi que selon le contexte culturel (Warde et al., 2007 ; Danesi, 2013 ; Gatley et al., 2014).

Des transformations importantes se sont opérées dans la contemporanéité affectant le rapport à l'alimentation et à l'activité culinaire. Les processus d'industrialisation et d'urbanisation, les avancées technologiques, les transformations des structures familiales et des rôles sociaux des hommes et des femmes, l'entrée de ces dernières dans la vie publique, les crises alimentaires, la médicalisation de l'alimentation, la montée de l'individualisme et de la réflexivité, etc., autant de mutations ayant transformé la nature de l'acte alimentaire et les modalités de sa mise en œuvre (Fischler, 1990 ; Mennell et al., 1993 ; Poulain, 2002a ; Contreras et GraciaArnaiz, 2005 ; Diaz-Méndez et Garcia-Espejo, 2014). Ce contexte est aussi marqué par des transformations spatio-temporelles, créant une tension entre le global et le local, entre globalisation et localisation (Giddens, 1991 ; Robertson, 1992 ; Canclini, 1997), entre uniformisation et diversification des normes, représentations et pratiques alimentaires et culinaires à l'échelle planétaire (Warde et al., 2007 ; Jourdan et Riley,

\footnotetext{
${ }^{4}$ Nous utiliserons également les expressions « faire la cuisine », « cuisiner » ou « acte culinaire » pour faire référence à l'activité culinaire.
} 
2013 ; Danesi, 2013).

De nombreuses recherches ont cherché à analyser les effets de ces phénomènes sur les modèles alimentaires (Herpin, 1988; Poulain, 2002b ; Barbosa, 2007, 2010 ; Carvalho et al., 2017), notamment afin de confronter la thèse de l'individualisation et de la déstructuration des pratiques alimentaires, diffusée à partir de la fin des années 1970, principalement à partir de la théorie de la «gastro-anomie » proposée par Fischler (1979). Leurs résultats attestent que malgré les transformations sociétales, les conventions socioculturelles jouent toujours un rôle important sur l'acte alimentaire. De plus, des tendances furent constatées à propos de l'activité culinaire : l'alimentation hors-foyer augmente alors que le temps consacré à la préparation culinaire diminue dans la majorité des pays industrialisés (Warde et al., 2007 ; Diaz-Méndez et Garcia-Espejo, 2014 ; Schubert et al., 2017); les produits préparés ou pré-préparés se sont progressivement introduits dans les foyers, entrainant l'exclusion d'étapes du processus culinaire et une modification de l'organisation du travail culinaire (Fischler, 1990 ; Warde, 1999 ; IBGE, 2011 ; Carvalho et al., 2017) ; en contrepartie, des études attestent que les individus valorisent de plus en plus la nourriture « cuisinée » et « faite maison » (Collaço, 2003 ; Mathé et al., 2014); enfin, de nouvelles disciplines corporelles et alimentaires sont apparues du fait du processus de médicalisation de l'alimentation (Poulain, 2002b) et du phénomène de la lipophobie (Fischler, 1990), favorisant ainsi l'émergence d'une cuisine nouvelle, la « cuisine light »(Santos, 2008).

La question de la diversité/uniformité culturelle de l'activité culinaire a pourtant été peu abordée, notamment en ce qui concerne la place accordée à cette activité dans le rapport à l'alimentation, ainsi que les significations qui lui sont attribuées. En effet, des travaux comparatifs ont analysé la diversité/uniformisation socioculturelle à partir d'autres dimensions des modèles alimentaires, tels que le rapport au goût ou au plaisir, la commensalité ou la notion de «bien manger » (Pfirsch, 1997 ; Rozin et al., 1999 ; Fischler et Masson, 2008; Shields-Argèles, 2012; Danesi, 2013). Par ailleurs, la majorité des études comparatives s'intéressant plus précisément à l'activité culinaire s'est basée sur des approches quantitatives, restreinte aux contextes européens et limitée à une analyse des pratiques culinaires, ainsi que du temps consacré à la préparation alimentaire (Rozin et al., 1999 ; Pettinger et al., 2006; Warde et al., 2007 ; DiazMéndez et Garcia-Espejo, 2014 ; Gatley et al., 2014). La compréhension du rapport à l'activité culinaire et des significations attribuées par différents groupes socioculturels à 
cette activité apparait donc comme une thématique à explorer, notamment dans la perspective des débats sur les transformations de l'alimentation dans la contemporanéité, ainsi que sur l'uniformisation et la diversification des normes, représentations et pratiques à travers le monde. Dans ce sens, en s'inscrivant dans cette tradition comparatiste et à partir d'une approche qualitative, nous cherchons à comprendre les façons de signifier et d'expérimenter l'activité culinaire de nos jours, au sein de deux pays présentant des modèles alimentaires différents : la France et le Brésil.

En France, pays mondialement reconnu pour sa gastronomie, l'importance accordée au plaisir de la table, au goût, à la convivialité, à l'activité culinaire et à la qualité des produits par rapport à leur origine et à leur mode de production, représente un aspect central du modèle alimentaire (Pfirsch, 1997 ; Rozin et al., 1999 ; Pettinger et al., 2006 ; Fischler et Masson, 2008 ; Mathé et al., 2009, 2014 ; TNS Sofres, 2014). Selon 1'Association Nationale des Industries Alimentaires (2015), 92\% des français estiment que l'alimentation est une composante essentielle du style de vie à la française. De plus, la singularité du modèle alimentaire français réside dans sa diffusion au sein des différentes couches sociales et dans son degré de complexité (Poulain, 2002a). L'inscription du «repas gastronomique des Français» sur la liste des patrimoines immatériels de l'humanité par l'UNESCO a par ailleurs renforcé ces dimensions chez les français (Masson, 2013). Au regard de ce contexte, de nombreux auteurs suggèrent que l'approche médicalisée de l'alimentation y progresse plus lentement que dans d'autres pays du monde (Rozin et al., 1999 ; Mathé et al., 2009 ; Shilds-Argelès, 2012).

$\mathrm{Au}$ Brésil, une vision hédoniste et conviviale de l'acte alimentaire (Barbosa, 2007) cohabite avec une perception fonctionnelle et médicalisée (Castro et al., 2014 ; TNS Sofres, 2014). Dans ce contexte, la cuisine était jusqu'à récemment un lieu méprisé et une activité perçue négativement, en tant que tâche domestique réservée aux femmes (Barbosa, 2010). En outre, des études révèlent une faible valorisation des aspects durables à propos de l'origine et des modes de production et de culture des produits (TNS Sofres, 2014; Galindo et Portilho, 2015; Azevedo, 2015). Il est également à souligner que la perception fonctionnelle de l'alimentation est accompagnée d'une valorisation significative de l'esthétique corporelle (Malysse, 2002 ; Sant'Anna, 2008). De plus, bien que le Brésil ait reçu des influences culturelles variées, il a aussi reçu (et reçoit encore) des influences considérables de la culture nordaméricaine, impactant sur le rapport à l'alimentation et au corps (Malysse, 2002 ; 
Sant'Anna, 2008 ; Santos, 2008). Il s'avère ainsi intéressant de comprendre le contexte brésilien en comparaison au contexte français, ce dernier se différenciant des Etats-Unis dans son rapport à la nourriture (Rozin et al., 1999 ; Fischler et Masson, 2008 ; ShieldsArgelès, 2012).

Cette approche comparative France-Brésil a été mise en place à partir de discours de femmes de nationalité française et brésilienne. En effet, les enjeux sur l'alimentation ont une influence différenciée sur les femmes (Fournier et al., 2015); la féminité implique de prendre soin d'autrui, notamment au travers de l'alimentation (Devault, 1991 ; Gracia-Arnaiz, 1996). La cuisine domestique quotidienne demeure une tâche effectuée principalement par les femmes, alors que la cuisine professionnelle (notamment la plus prestigieuse) est surtout assurée par les hommes (Barbosa, 2007 ; Collaço, 2008 ; Marie, 2014 ; Diaz-Méndez et Garcia-Espejo, 2014).

Dans cette étude nous cherchons donc à comprendre comment des femmes issues de deux nationalités distinctes, marquées par des contextes socioculturels et modèles alimentaires différents, signifient et expérimentent l'activité culinaire. Nous partons du postulat que s'il existe une certaine standardisation des pratiques et normes alimentaires et culinaires à travers le monde, des phénomènes d'ordre global, exogènes aux cadres nationaux et propres à la contemporanéité, sont incorporés, expérimentés et redéfinis différemment au sein de chaque réalité sociale - historiquement, culturellement et spatialement localisée - à partir de leurs normes socioculturelles, leurs caractéristiques environnementales et socio-économiques (Warde et al., 2007 ; Danesi, 2013). Les interrogations suivantes ont orienté l'étude: comment l'activité culinaire est expérimentée et signifiée au quotidien par des femmes françaises et brésiliennes ? Quels sont les éléments qui rapprochent ou différentient le rapport au culinaire des femmes de ces deux nationalités ? En quoi les singularités des modèles alimentaires influencent la façon de signifier et d'expérimenter l'acte culinaire ? Les données recherchées ont pour objectif d'enrichir les débats autour des modèles alimentaires dans un contexte de changements sociétaux profonds. Par ailleurs, elles cherchent à élargir les débats sur l'uniformisation et la diversification des normes, représentations et pratiques alimentaires à l'échelle planétaire.

\section{Méthodologie}


L'étude se base sur une méthodologie qualitative. Bien qu'il s'agisse d'une méthodologie peu utilisée dans les études sur l'activité culinaire, elle se montre plus adéquate pour une meilleure compréhension des expériences et significations associées à cette activité (Short, 2003). Une méthodologie comparative internationale synchronique fut également adoptée. D’après Warde et al. (2007), cette approche est intéressante afin de comprendre la multiplicité des facteurs pesant sur l'acte alimentaire. La comparaison entre des discours de différents groupes socioculturels peut également dégager, d'une part, ce qui est commun entre des pays ou régions distinctes et propre à notre époque et, d'autre part, ce qui est spécifique à chaque culture (Fischler et Masson, 2008). Bien que nous ne considérons pas que les pays correspondent à un ensemble figé et homogène d'individus, effectuer une comparaison sur l'alimentation entre deux ou plusieurs pays, semble être une approche pertinente. Au-delà des différences qui peuvent exister entre les membres d'un même pays selon les différentes variables sociales,

nous pensons que notre vision du monde - disons notre façon d'imaginer le monde et notre place en son sein en termes de 'eux' et de 'nous' et de 'maintenant' et 'alors' - est en grande partie façonnée par la nation à laquelle nous appartenons, et que cette vision du monde se transmet et se maintient non seulement par un certain nombre d'institutions ou rituels nationaux, mais aussi à travers une multitude d'objets et de pratiques du quotidien (Edensor, 2002) (Shields-Argelès, $2012: 24$ ).

L'alimentation est une pratique quotidienne qui participe à la construction des identités sociales (Fischler, 1990 ; Poulain, 2002a). Les normes familiales et culturelles, les États providence et les institutions publiques façonnent des styles et des modèles alimentaires au sein du cadre national (Danesi, 2013). Les conditions et les situations alimentaires sont donc différentes au sein des différents pays où des normes socioculturelles distinctes y influencent la façon de manger (Fischler et Masson, 2008). Danesi (2013) affirme que le pays est un cadre d'analyse pertinent et incontournable dans le domaine de l'alimentation, étant donné qu'il fournit un ensemble de repères faisant partie intégrante du quotidien de ses citoyens, constituant un contexte institutionnel au sein duquel la population se socialise.

Les unités de comparaison ont été déterminées à partir d'un choix pragmatique, de par notre familiarité avec les groupes étudiés, et d'un choix stratégique, selon la problématique de l'étude (Vigour, 2005). La recherche a été conduite dans des zones urbaines: Toulouse en France et São Paulo au Brésil. Il est à noter que bien que certaines informatrices étaient originaires d'autres villes, elles résidaient à Sao Paulo ou à Toulouse au moment de l'entretien. Au-delà de la nationalité, la population cible fut 
définie selon le genre, l'âge, la profession ou le niveau d'étude. Cet article comprend des résultats d'une étude doctorale portant sur les normes et pratiques alimentaires de diététiciennes et femmes profanes françaises et brésiliennes. Les informatrices font ainsi partie de ces deux groupes :

(i) les diététiciennes. La profession de diététicien est composée essentiellement de femmes (Sicart, 2014). Une diététicienne est doublement marquée par les enjeux de l'alimentation: d'une part, elle est une femme, un être «social», concernée par ces enjeux dans sa vie personnelle; d'autre part, elle travaille autour de ces enjeux avec ses patients. L'étude est limitée aux diététiciennes travaillant en libéral, sans distinction d'âge ;

(ii) les jeunes femmes profanes vis-à-vis de la diététique, groupe composé d'étudiantes à l'université. Nous avons cherché l'hétérogénéité de l'échantillon par rapport aux disciplines étudiées par ces femmes, ainsi que le type d'établissement (public ou privé). Nous avons exclu les étudiantes en Nutrition Humaine et Diététique. Les femmes interviewées étaient âgées de 18 à 30 ans, période caractérisée par la construction de l'autonomie, y compris alimentaire. Dans ce contexte, l'alimentation devient un lieu de revendication d'une identité générationnelle (Garabuau-Moussaoui, 2001).

Bien que nous ayons défini des critères précis dans le choix des unités de comparaison, il faut souligner que le choix des contextes d'étude ne s'est pas fait en les considérant comme des réalités parfaitement symétriques les unes par rapport aux autres. La symétrie des cas analysés dans une comparaison est en effet une problématique qui interpelle les chercheurs engagés dans des études comparatives et qui mérite une attention particulière. « Toute comparaison est-elle possible ? » se demande Vigour (2005 : 153) dans son ouvrage sur l'approche comparative en sciences sociales. D'après l'auteur, quel que soit l'objet sélectionné, la comparaison révèle toujours des différences et ressemblances. La problématique de la symétrie se pose également quant au dispositif d'enquête (Verdalle et al., 2012). Comme le suggère Boas (2011), la démarche comparative n'est pas toujours heuristique en fonction des contextes, objets et phénomènes d'analyse. Ainsi, les réalités observées dans cette étude ne sont pas symétriques les unes par rapport aux autres. À titre d'exemple, appartenir à la classe moyenne au Brésil n'est pas équivalent à appartenir à la classe moyenne en France, de même que concernant les classes sociales aisées ou défavorisées, qui ne présentent pas 
les mêmes caractéristiques et conditions d'un contexte à l'autre. Dans la présente étude, les informatrices appartiennent aux classes moyenne ou aisée de leur pays respectif, et c'est ainsi à partir de cette observation que nous nous proposons de comparer les informatrices des deux nationalités, c'est-à-dire sans considérer la symétrie d'un pays à l'autre. En outre, bien que Toulouse et São Paulo soient des zones urbaines vastes et importantes au sein de leur pays respectif, elles présentent des différences entre elles. Il est en effet difficile de comparer l'expérience de vie urbaine toulousaine avec celle d'une mégalopole comme São Paulo. De la même façon, être une jeune femme, une étudiante à l'université ou une diététicienne en France et au Brésil révèle également une asymétrie. Dans cet article nous ne considérons pas ces asymétries comme une limitation. En effet, la collecte de données dans des contextes asymétrique, lorsqu'elle est incorporée à l'analyse et à l'écriture de la comparaison, constitue moins un obstacle à la comparaison qu'un processus qui renseigne lui-même sur les cas étudiés et sur leurs différences (Boas, 2011 ; Verdalle et al., 2012).

Le travail de terrain a été effectué de 2012 à 2015 au travers d'observations « flottantes » (Pétonnet, 1982) réalisées au cours de la vie quotidienne et principalement au travers d'entretiens individuels semi-directifs. L'entretien est pertinent lors de l'analyse des significations données aux pratiques par les acteurs sociaux, ainsi que des valeurs et systèmes normatifs sur lesquels ces pratiques se fondent (Blanchet et Gotman, 2005). Les entretiens ont été orientés par un guide d'entretien en français et en portugais. Lors des entretiens, nous avons utilisé des images d'aliments afin de mettre en évidence les rationalités mobilisées lors de la réflexion autour d'une même image. Nous avons ainsi présenté des images de légumes, de fruits, de produits allégés, de viande de bœuf et d'un fondant au chocolat accompagné d'une glace. Des aliments faisant partie du répertoire culturel ont également été présentés : la baguette, le fromage et le cassoulet en France ; la « feijoada » et le « pão de queijo » au Brésil.

Quinze entretiens ont été réalisés dans chaque pays auprès des diététiciennes. La recherche des diététiciennes fut réalisée : (i) sur internet, à partir de mots clés saisis sur Google (par exemple, «Diététicienne à Toulouse ») ; (ii) au travers de l'entourage des chercheurs; (iii) par la méthode «boule-de-neige», certaines diététiciennes interviewées nous ayant mis en contact avec d'autres informatrices. Les professionnelles furent contactées par email ou téléphone. Après leur avoir rapidement présenté l'objet de l'étude, nous leur avons demandé leur disponibilité en vue d'un 
entretien. Les entretiens, d'une durée de 40 minutes à deux heures, furent majoritairement réalisés au sein de leur cabinet. Quant aux jeunes femmes, 25 entretiens ont été réalisés au Brésil et 29 en France. La recherche de ces jeunes femmes s'est faite de manière aléatoire sur les campus universitaires. De plus, des contacts ont été obtenus à partir de l'entourage des chercheurs, ainsi qu'au travers de la méthode «boule-deneige ». Les femmes furent d'abord informées des objectifs de l'étude, puis nous leur avons demandé leur disponibilité en vue d'un entretien. Les entretiens, d'une durée de 40 minutes à deux heures, se sont majoritairement déroulés au sein des universités ou dans des cafés/bars choisis par les informatrices.

Les entretiens furent enregistrés sous l'accord des informatrices. Leur retranscription intégrale a permis de dégager de premières constatations et thématiques récurrentes. Nous avons ensuite effectué des analyses individuelles par informatrice et des analyses générales par nationalité, à partir des tableaux thématiques et de catégories. Nous avons effectué un choix méthodologique et analytique pour la construction du travail. En effet, l'ensemble des thématiques évoquées par les informatrices s'est retrouvé chez les deux nationalités. Nous avons donc abordé les thématiques et les catégories de façon générale en pointant du doigt les particularismes de chaque nationalité ou groupe. Cette approche nous permet non seulement de rester fidèle à la réalité sociale observée sur le terrain, mais aussi d'éviter de cristalliser des stéréotypes (Vigour, 2005). Par ailleurs, il est à noter que bien que nous nous référons aux informatrices à partir de leur nationalité, les résultats ne peuvent pas être généralisés à toutes les diététiciennes et jeunes femmes françaises et brésiliennes, car il s'agit de contextes spécifiques d'un point de vue géographique, social, de l'activité exercée par les diététiciennes et du niveau d'éducation.

Les résultats seront abordés à travers trois parties principales: (i) l'activité culinaire, un lieu féminin? ; (ii) la place de l'activité culinaire : des discours aux pratiques $^{5}$; (iii) les significations de l'activité culinaire.

\footnotetext{
${ }^{5}$ Nous allons aborder les pratiques des informatrices à partir de données concernant leurs «pratiques déclarées ", c'est-à-dire à partir de ce que les informatrices déclarent avoir fait (Poulain, 2002b). Ces pratiques déclarées peuvent ne pas représenter la réalité et être influencées par les systèmes normatifs. En effet, il existe des croyances qui collaborent à la construction des critères dans l'évaluation de ce qui est correct ou incorrect dans une perspective sociale, et qui conditionnent les réponses que les sujets donnent lorsqu'ils décrivent ce qu'ils mangent. Ces pratiques déclarées font donc l'objet de transformations, de restructurations sémantiques, d'oublis, de négociations ou d'occultations. Ces discours sont pourtant importants car ils révèlent la façon dont les acteurs sociaux organisent leurs connaissances et expériences alimentaires au travers du langage, comment ils les perçoivent et les expriment (Poulain, 2002b ; Contreras et Gracia-Arnaiz, 2005).
} 


\section{Résultats}

\section{L’activité culinaire, un lieu féminin ?}

L'analyse des discours des informatrices des deux nationalités concernant l'activité culinaire a mis en évidence l'omniprésence des figures féminines. En effet, un de nos objectifs était d'identifier dans le foyer la personne en charge de cuisiner, ainsi que de comprendre le processus d'apprentissage culinaire. Les discours des informatrices montrent que l'activité culinaire reste surtout à la charge des femmes. Qu'il s'agisse des informatrices elles-mêmes, de leur mère, de leurs grand-mères ou des femmes de ménage, la préparation alimentaire ou le choix des menus, notamment au quotidien, est assurée par les figures féminines du foyer. En effet, dans la majorité des cas, leur père ou leur compagnon peut collaborer, surtout lors des étapes en amont de la préparation, tel que l'achat des produits alimentaires. Par ailleurs, s'il arrive qu'ils participent à la préparation, ils sont généralement conseillés par des femmes ou assument cette responsabilité occasionnellement lors des weekends ou contextes festifs. Ces observations permettent de confirmer la permanence de certaines dynamiques sociales autour de l'activité culinaire: les femmes sont davantage en charge de la cuisine domestique, alors que les hommes s'occupent de la cuisine plutôt dans les occasions festives.

Devault (1991), dans son ouvrage Feeding the Family: The Social Organization of Caring as Gendered Work, observe que les femmes continuent à être les plus impliquées de la préparation alimentaire au sein du foyer et que cette tâche ne représente pas seulement une activité pratique, mais aussi un acte qui fonctionne symboliquement en construisant les compréhensions à propos de la féminité et de la famille. D'après Devault, l'activité culinaire peut être gratifiante et significative pour les femmes, mais elle peut néanmoins s'avérer pénible et oppressante, reléguant les femmes à un statut secondaire au sein du foyer. En effet, le partage différentiel de cette tâche provoque une asymétrie qui renforce l'inégalité de genre. Gracia-Arnaiz (1996) souligne que si la différentiation entre cuisine quotidienne (féminine) et cuisine spécialisée (masculine) sert, à priori, à mettre en question les aspects biologiques de certaines attributions, il lui paraît nécessaire de considérer que la responsabilité féminine envers l'alimentation quotidienne est associée à une transmission « naturelle » des tâches domestiques aux femmes et, notamment, des tâches liées au soin des 
membres de la famille. Cette responsabilité «naturelle» peut être résumée dans l'obligation féminine de nourrir les différents membres du groupe, d'offrir les aliments prêts à la consommation. Bien que cet acte puisse, tout d'abord, être interprété comme une responsabilité de satisfaire des besoins physiologiques, il comporte également la reproduction et la satisfaction d'autres dimensions sociales.

La transmission culinaire est aussi marquée par la présence féminine. Bien que les informatrices indiquent un apprentissage culinaire individuel (guidé par des contenues présents dans les magazines, internet ou ouvrages), lorsqu'elles mentionnent une personne responsable de cet apprentissage il s'agit systématiquement d'une figure féminine: la mère ou la grand-mère. La transmission de connaissances culinaires s'opère de façon explicite et formalisée, mais aussi de façon indirecte, au travers de l'observation et l'aide aux préparations culinaires. Comme l'observent également Loisel et Couvreur (2004 : 70) auprès de femmes françaises, « on apprend et on transmet un peu malgré soi ». De plus, les informatrices considèrent souvent leur mère comme une source d'information fiable en matière d'alimentation et comme le membre de la famille responsable de leur éducation alimentaire. Garabuau-Moussaoui (2001) a aussi constaté auprès de jeunes français que la mère est une source de savoir-faire culinaire et une référence positive de l'activité culinaire. Ceci révèle que malgré les transformations opérées dans les dernières décennies et l'émergence de nouvelles formes de transmissions des savoir-faire culinaires, cette transmission demeure effectuée principalement à partir de la figure féminine. Par ailleurs, ces observations témoignent qu'il persiste de nos jours des transmissions alimentaires intergénérationnelles. Lorsque les informatrices (peu nombreuses et surtout françaises) attribuent à leur père leur apprentissage culinaire, celui-ci exerce, dans la majorité des cas, une activité professionnelle liée à la cuisine, comme boucher ou traiteur.

Malgré la constatation d'une implication générale des femmes dans l'activité culinaire, la place accordée à cette dernière et son expérience quotidienne est diverse chez les informatrices.

\section{La place de l'activité culinaire : des discours aux pratiques}

L'activité culinaire est expérimentée différemment selon l'informatrice, pouvant ainsi singulariser les trajectoires de vie de chacune. Par ailleurs, il existe des disparités entre les deux nationalités. Lors des entretiens, les réponses aux questions sur l'activité 
culinaire montrent combien elles sont impliquées dans cette thématique. En général, au Brésil, les réponses sont rapides. En France, les réponses montrent davantage d'enthousiasme et présentent un contenu plus riche. De plus, les françaises sont plus nombreuses à déclarer savoir cuisiner. L'analyse de l'ensemble des discours des informatrices confirme ces différences. Par exemple, à la vue des images d'aliments montrées lors des entretiens, les françaises évoquent, plus que les brésiliennes, des aspects culinaires, notamment lorsqu'il s'agit de légumes :

Les légumes, c'est un aliment sain pour moi et je prends du plaisir à cuisiner, donc c'est sympa à travailler (Morgane ${ }^{6}$, française, 21 ans).

Par ailleurs, lorsque les informatrices donnent leurs avis sur l'alimentation de leur pays, les françaises mentionnent davantage la dimension culinaire. En outre, cuisiner est un acte davantage présent dans le rapport que les françaises entretiennent avec leur alimentation et dans la valeur qu'elles lui accordent. En effet, la thématique alimentaire est indissociable de l'aspect culinaire chez les françaises.

Nous avons effectué une typologie des informatrices selon le rapport entretenu à l'activité culinaire: (i) les informatrices parlant beaucoup de la cuisine, cuisinant fréquemment et aimant cette activité ; (ii) les informatrices accordant peu d'attention à la thématique de l'activité culinaire, cuisinant de temps en temps, mais aimant tout de même cette activité ; (iii) les informatrices parlant peu de la cuisine, ne cuisinant pas et percevant l'activité culinaire comme une contrainte. Nous constatons qu'en France elles sont plus nombreuses à correspondre aux deux premières typologies, tandis qu'au Brésil les deux dernières typologies sont plus représentatives des informatrices.

Le fait que les discours des françaises soient davantage imprégnés de la thématique de l'activité culinaire pourrait être associé à la place qu'elle occupe dans leur quotidien. Au Brésil, parmi les 40 femmes interrogées, dix déclarent cuisiner fréquemment, sept parfois et 23 rarement ou jamais. Parmi ces dernières, huit font réaliser cette activité par une femme de ménage, 11 déclarent que c'est leur mère qui cuisine et deux mangent à l'extérieur ou des plats déjà préparés (information non disponible chez deux informatrices). Silvia (diététicienne brésilienne, 38 ans) cuisine rarement. Afin de l'illustrer, elle évoque le nombre de sacs de riz, aliment de base de la cuisine brésilienne (Barbosa, 2007), qu'elle a cuisinée au cours des cinq dernières années :

\footnotetext{
${ }^{6}$ Les prénoms des informatrices ont été modifiés afin de préserver leur anonymat.
} 
Je ne cuisine pas, je ne suis pas une amoureuse de la cuisine. Pour que tu puisses comprendre, depuis ces cinq années où je suis mariée, on est dans le deuxième sac de riz de 5 kilos.

Les discours des françaises révèlent un autre scénario. Sur les 44 françaises interviewées, la majorité (33) déclare cuisiner fréquemment, tandis que 3 le font parfois et 8 rarement ou jamais. Par ailleurs, plusieurs françaises racontent qu'elles préparent elles-mêmes leur propre pain et yaourt. L'activité culinaire comme expérience quotidienne n'est donc pas vécue de façon similaire suivant les informatrices et nous révélons des disparités selon la nationalité.

Le départ du domicile parental et l'émancipation pourraient avoir une influence. En France, cinq informatrices habitent chez leurs parents, alors que chez les brésiliennes, la tendance est presque inverse : 22 informatrices habitent chez leurs parents. Le départ du domicile parental et l'émancipation sont associés aux conditions sociales, économiques, politiques et culturelles de chaque pays. Selon l'Eurostat, l'âge moyen de départ du domicile parental en France était en 2013 de 23,6 ans (Commission Européenne, 2015). Au Brésil, selon des données de l'IBGE publiées le 21 janvier 2016 dans la revue Istoé, l'âge moyen de départ du foyer familial était de 35 ans. Ce processus pourrait augmenter la responsabilité vis-à-vis de l'activité culinaire, comme il l'est souligné par certaines informatrices. Pourtant, en France elles sont plus nombreuses à avoir été initiées à cette activité depuis leur plus jeune âge, révélant ainsi que d'autres éléments pourraient jouer sur la place qu'occupe l'activité culinaire dans leur vie.

Une conversation informelle avec un français de 23 ans, issu d'une famille aisée, a attiré notre attention. Nous lui avons demandé s'il savait cuisiner et il a répondu : «Bien sûr que je sais cuisiner, est-ce tu connais quelqu'un qui ne sait pas cuisiner ? ». Notre question l'a étonné alors qu'elle nous semblait, étant plus familiarisés avec le contexte brésilien, tout à fait légitime. Nous nous sommes donc rendus compte que nous avions affaire à deux univers distincts dans la façon d'expérimenter l'activité culinaire. Cette question posée à un brésilien ne susciterait probablement aucun étonnement et la réponse pourrait même être négative, tel nous avons nous constaté auprès de nos informatrices.

Lors de l'entretien, Flor (brésilienne, 26 ans) explique que lorsque la femme de ménage de ses parents n'a plus dormi chez eux, ils ont alors changé leurs pratiques, car «il n'y avait personne pour cuisiner ». Nous comprenons ainsi que pour sa famille cuisiner n'est pas envisageable au quotidien, car cette tâche revenant à la femme de 
ménage. Sachant que les informatrices brésiliennes sont issues des classes moyennes et aisées, cette présence moins marquée de l'activité culinaire dans leurs discours et leurs pratiques quotidiennes pourrait être associé à un aspect socioculturel, économique et politique du pays. Héritage d'une société esclavocrate, les rapports familiaux des maisons brésiliennes des classes moyennes et aisées sont marqués par la présence de serviteurs. Ils s'affairent aux tâches domestiques, l'activité culinaire (comme d'autres activités) étant ainsi en grande partie transférée à la femme de ménage (Brites, 2007 ; Collaço, 2008). Bien que les informatrices brésiliennes valorisent le fait que la nourriture soit cuisinée dans le foyer, il s'agit d'une activité peu fréquente au quotidien et souvent effectuée par les femmes de ménages. Par ailleurs, Barbosa (2010) suggère que jusqu'à récemment la cuisine portait une valeur négative et était associé à un lieu méprisé, surtout destiné aux femmes dans le travail domestique. Dans la maison brésilienne moderne, héritière des demeures coloniales et construite selon des « espaces de ségrégation »(Brites, 2007), la cuisine (en tant que lieu de la maison) est généralement rattachée à l'axe le moins prestigieux, celui de « service » (Gomes, 2016). Il s'agirait ainsi d'une activité non seulement assurer davantage par les femmes de ménage, mais aussi associée à une tâche socialement et symboliquement dévalorisante. Le fait de « devoir » cuisiner au quotidien (ou effectuer d'autres tâches domestiques manuelles) pourrait être considéré comme un «marquage social dévalorisant» (Corbeau, 2002).

Alors que les femmes de ménage ne sont jamais évoquées par les françaises, elles sont fréquemment mentionnées par les brésiliennes. Par ailleurs, nos observations dans les deux pays confirment ces différences concernant la présence des femmes de ménages dans les foyers. Bruna (brésilienne, 24 ans) habite chez ses parents où c'est une femme de ménage qui cuisine. Elle critique sa façon de cuisiner :

La femme de ménage qui travaille chez nous ajoute trop d'huile dans la nourriture, je n'aime pas, je ferais différemment.

Les diététiciennes vont jusqu'à adapter leurs services à ce système. En effet, elles proposent une formation des femmes de ménage afin qu'elles cuisinent « sainement » :

Une diététicienne va chez le patient, amène un menu, donne des orientations à la femme de ménage, sur la quantité d'huile à utiliser, comment cuisiner pour ne pas perdre de nutriments (Fabiana, diététicienne brésilienne, 31 ans).

Juliane (diététicienne brésilienne, 27 ans) a suivi une formation en gastronomie afin d'offrir ce service. Elle explique qu'il peut exister un conflit entre la diététicienne et la femme de ménage qui aurait plus d'expérience dans la cuisine : 
Il y a des employées qui adorent l'idée, car c'est un investissement de la patronne envers elles, on donne un certificat (...) il y en a d'autres qui disent 'je sais tout, je travaille depuis 30 ans dans la cuisine, j'ai déjà travaillé dans mille restaurants, qu'est-ce qu'une jeune fille va m'apprendre?' Il y a plus de résistance. C'est embêtant, car le patient m'a contracté pour lui apprendre de nouvelles recettes, l'aspect de la qualité, tel qu'utiliser moins de sel et de graisse.

Dans ce contexte, bien que généralement la femme de maison (l'employeur) fasse les courses alimentaires et donne des directives de réalisation culinaire à la femme de ménage, de nombreux brésiliens, notamment issus des classes moyennes et aisées, ne cuisinent pas et/ou n'apprennent pas à cuisiner. Ce système peut influencer le rapport que les individus, comme certaines de nos informatrices, entretiennent envers l'activité culinaire et l'alimentation de façon plus générale. Ils maintiennent une distance vis-àvis de cette activité, notamment dans le cadre du quotidien. Il est pourtant à noter une valorisation récente de l'activité culinaire et de nouvelles formes de socialisation de la cuisine, surtout au sein des classes sociales aisées (Barbosa, 2010).

Dans le cas des françaises, leur attachement à l'activité culinaire pourrait être en partie lié aux caractéristiques de leur modèle alimentaire. La France est un des pays au monde qui s'(auto)représente le plus au travers de la gastronomie et de l'activité culinaire. En effet, la France est connue comme le pays de la gastronomie (Poulain, 2002a ; Fischler et Masson, 2008) et les français sont socialisés en intériorisant cette idée. Par exemple, lorsque Laurianne (diététicienne française, 28 ans) parle du rapport entre santé et plaisir :

Je suis entre les deux, parce qu'on est en France. La gastronomie, en France, c'est quelque chose de conviviale, et depuis que je suis petite le temps des repas c'est un temps très important dans ma famille, et chez moi c'est pareil. Le moment du repas c'est important et pour moi c'est un plaisir.

Comme nous l'avons remarqué, le modèle alimentaire français est marqué par l'importance accordée au plaisir, au goût, à la convivialité, à l'activité culinaire et à la qualité des produits (Rozin et al., 1999 ; Fischler et Masson, 2008 ; Mathé et al., 2009, 2014 ; Shilds-Argelès, 2012 ; TNS Sofres, 2014). Shields-Argèles (2012), en comparant les représentations sociales du «bien manger» chez des français et des nordaméricains, vérifie des particularités dans les discours des français qu'elle désigne comme un "discours gastronomique », caractérisé par l'importance du plaisir, de la commensalité, la valorisation des produits et de l'activité culinaire. Poulain (2002a) remarque que cette attitude gastronomique représente un patrimoine revendiqué avec fierté par tous ceux qui sont partie prenante de la culture française. Ce discours est aussi vérifié par d'autres auteurs (Rozin et al., 1999 ; Pfirsch, 1997 ; Mathé et al., 2009). En s'appuyant sur une enquête réalisée par le CSA en 1987 montrant que pour $63 \%$ des 
français l'identité nationale est symbolisée par la cuisine, Drouard (2005) affirme que la cuisine est une des expressions les plus fortes de l'identité française. L'inscription du « repas gastronomique des Français» sur la liste du patrimoine immatériel de l'humanité de l'UNESCO a probablement renforcé cette association entre identité française et le culinaire. En effet, cette candidature a suscité un intérêt public et fait l'objet d'une importante médiatisation. D'après Masson (2013), l'inscription à l'UNESCO fut pour beaucoup de français une source de fierté, notamment parce qu'ils reconnaissent dans cette pratique un ensemble de rituels et un système de valeurs qui leur tient à cœur. Mathé et al. (2009) ont vérifié, par exemple, que 84\% des français conçoivent «le repas gastronomique» surtout comme « une culture dont tous les français sont porteurs » et moins comme « une affaire de grand chef» $(69 \%)$.

Ces différences entre les informatrices brésiliennes et françaises concernant la place occupée par l'activité culinaire s'accompagnent de significations distinctes attribuées à cette pratique.

\section{Les significations de l'activité culinaire}

Les discours des informatrices permettent de constater que l'activité culinaire est signifiée de diverses façons. Nous avons regroupé les significations associées à l'activité culinaire par les informatrices françaises et brésiliennes en quatre catégories : « activité culinaire pratique et contraignante »; « activité culinaire comme construction de soi »; «activité culinaire comme partage et intégration»; «activité culinaire comme source de plaisir ». Ces significations intègrent des éléments qui vont du matériel au symbolique et dépendent des circonstances sociales, temporelles, financières et spatiales. Les significations observées ne sont pas des catégories dichotomiques, leurs frontières sont parfois floues et il arrive qu'elles se superposent. En outre, elles peuvent être plus ou moins latentes selon les contextes et les moments de la vie. Ces résultats témoignent du dynamisme et de la pluralité de l'acte alimentaire, résultat de l'interaction entre mangeur, aliment et situation (Corbeau, 2002). Par exemple, il existe une différence entre les logiques d'action orientant les pratiques alimentaires/culinaires du lundi au vendredi et celles du weekend/occasion festive.

$\underline{\text { Activité culinaire pratique et contraignante }}$ 
Cette catégorie regroupe les idées associées à une activité culinaire marquée principalement par l'aspect pratique et la vitesse. Elle est, dans ce cas, perçue comme une activité quotidienne parmi toute une série d'autres activités qui ne doit pas prendre beaucoup de temps. Elle est ainsi perçue comme une perte de temps. Alexandrine (française, 20 ans) habite seule et en plus d'étudier, travaille dans un supermarché. Elle cuisine toujours vite :

C'est sympa (cuisiner), mais pas des trucs compliqués. Non... je ne fais pas trop la cuisine. Il faut que ça soit rapide vu que je rentre à 10 heures le soir.

Manger est un besoin vital dont l'individu ne peut pas se passer, malgré le manque de temps et/ou d'envie de manger ou de cuisiner. Dans cette perspective, l'activité culinaire est essentiellement centrée sur l'aspect nourrissant et fonctionnel de l'alimentation. Il faut faire quelque chose « vite », « basique », « simple », afin d'avoir une préparation consommable et acceptable pour combler la faim. Faire à manger peut même devenir une contrainte, une tâche pour laquelle les informatrices font preuve de paresse et qui les empêche de se concentrer sur d'autres aspects de leur vie qu'elles aimeraient privilégier. Jana (brésilienne, 25 ans) écrit son mémoire de master et préfère travailler que faire à manger :

Je ne cuisine pas, parce que j'ai la flemme. Je ne veux pas m'arrêter de travailler pour faire à manger.

Helena (diététicienne brésilienne, 27 ans) cuisine rarement, il s'agit d'une tâche fatigante pour elle :

Je cuisine peu, comme je t'ai dit, je prends des pâtes, une boîte de thon et je mange plutôt ça que faire des choses délicieuses, la cuisine c'est quelque chose qui me fatigue.

Il s'agit d'un rapport plus associé aux jours de la semaine où les informatrices doivent accomplir d'autres responsabilités, comme le travail et/ou les études. Cette attitude est davantage présente chez les jeunes femmes et les brésiliennes de façon plus générale. En ce qui concerne les jeunes femmes, cette attitude semble être une caractéristique d'une étape de la vie. De façon générale, seulement 23,6\% des français considèrent la cuisine comme un corvée, mais c'est notamment chez les individus ayant moins de 34 ans que la perception de l'activité culinaire en tant qu'une obligation est plus forte (Poulain, 2002b). Par rapport à la différence entre les deux pays, il semble exister un paradoxe, car les brésiliennes sont celles qui assurent le moins la tâche culinaire au quotidien, mais sont pourtant celles qui évoquent plus fréquemment cette activité comme une contrainte. Lorsque nous demandons aux brésiliennes si elles cuisinent, certaines expliquent directement comment elles s'organisent dans la semaine 
pour optimiser leur temps, afin de limiter le temps consacré à la cuisine du lundi au vendredi. Les technologies alimentaires, comme le fait de conserver les préparations au congélateur, sont mises en place pour optimiser le temps culinaire-alimentaire.

\section{$\underline{\text { Activité culinaire comme construction de soi }}$}

Cette catégorie regroupe les significations désignant l'activité culinaire comme un acte de construction du soi, marqué par un enjeu corporel-physiologique, ainsi que par une attitude plutôt individuelle. Dans ce cas, l'activité culinaire permet la modification des aliments pour le façonnement du corps, de la forme physique et/ou de la santé. L'activité culinaire comme un acte de production de préparations perçues comme « saines » et «nutritionnellement adéquates » est évoquée par les deux nationalités, mais cette perception est davantage marquée chez les brésiliennes. Dorote (diététicienne brésilienne, 24 ans) précise son rapport à l'activité culinaire :

J'adapte les recettes pour que les plats soient plus sains, mais chez moi personne ne veut avoir le travail de cuisiner.

Il s'agit d'une perspective médicalisée, adossée à une rationalité scientifiquenutritionnelle, dans laquelle les effets physiologiques sont au premier plan. En effet, de façon générale, les discours des brésiliennes sont davantage imprégnés d'une vision nutritionnelle et fonctionnelle de l'alimentation, révélant l'importance accordée à ces dimensions. Ce rapport à l'alimentation est, par ailleurs, accompagné d'une préoccupation vis-à-vis de l'esthétique corporelle et des transformations corporelles volontaires, attitude moins prononcée chez les françaises.

Cette recherche d'une cuisine «saine" s'organise surtout autour des légumes, viandes blanches, des produits complets et allégés, mais aussi autour de techniques culinaires évitant l'apport de matières grasses, notamment d'origine animale. Morgane (française, 21 ans) essaie de cuisiner avec moins de beurre :

J'ai des parents qui cuisinent avec beaucoup de beurre, et le beurre j'évite parce qu'avec le cholestérol, je cuisine avec de l'huile surtout.

La préparation culinaire est aussi utilisée afin de maîtriser les micronutriments. Rosana (diététicienne brésilienne, 33 ans) décrit le «bien manger» en soulignant les aspects nutritionnels de la préparation d'une sauce tomate :

C'est avoir des aliments qui sont riches en nutriments, nécessaires pour l'organisme, les oligoéléments, les vitamines, les minéraux, les composants fonctionnels qui préviennent les maladies. Je pense à la préparation d'une sauce et au contrôle du lycopène. Je pense à la présence d'huile d'olive, de graisse mono insaturée (...).

Il s'agit aussi d'une vision pharmacologique de l'alimentation, où les frontières entre aliment/ingrédient et nutriment/médicament sont floues. Les recettes sont conçues 
à partir d'aliments choisis selon leur composition nutritionnelle et/ou à partir de compléments alimentaires. Par exemple, Raquel (diététicienne brésilienne, 30 ans) cuisine avec le complément nutritionnel Whey Protein et des blancs d'œufs lyophilisés, des produits de la "cuisine fitness » au Brésil. Les réseaux sociaux des diététiciennes, surtout brésiliennes, présentent des recettes où ingrédients et nutriments se confondent, comme nous l'observons dans le sous-titre d'une préparation postée par Luna (diététicienne brésilienne, 27 ans) : «Protéine à digestion facile + fibres + antioxydants + indice glycémique bas + facile à préparer \#cibledanslasanté ».

L'activité culinaire devient un outil sur lequel les diététiciennes s'appuient afin d'améliorer l'ingestion nutritionnelle de leurs patients. Comme le suggère Santos (2008), le travail culinaire migre du champ du savoir empirique et artisanal vers le champ scientifique. L'apparition de nouvelles disciplines corporelles et alimentaires implique une nouvelle cuisine, s'inscrivant dans un système de codification qui implique de nouvelles normes, de nouveaux critères diététiques et gustatifs de classification et sélection des produits, ainsi qu'un choix différentié de techniques de préparation culinaire. Cette cuisine rompt souvent avec les structures d'actions inscrites dans les traditions (Santos, 2008), s'opposant parfois à la cuisine familiale. Dans cette perspective, notamment au Brésil, des pratiques et préparations perçues comme 《 traditionnelles », telles que la « feijoada» ou la « caipirinha », sont (re)créées à partir de la substitution de certains ingrédients par d'autres perçus comme plus « sains » et/ou adaptées à partir de produits allégés/édulcorants.

Au-delà de la valeur nutritionnelle, certaines informatrices associent l'activité culinaire à une recherche de santé à partir d'autres paramètres qui échappent à une expérience individuelle nutritionnelle. Dans cette perspective, cette «cuisine saine» s'appuie, notamment chez les françaises, sur un contrôle de la qualité des produits en ce qui concerne leur origine et les processus de production et distribution. Dans ce cas, l'acte culinaire permet au mangeur de se sentir davantage intégré dans la filière alimentaire, se rassurant quant à ce qu'il ingère. Cuisiner permettrait de «travailler » des aliments naturels, frais, locaux, « de base», produits valorisés par ces informatrices :

(Manger équilibré) c'est travailler des aliments simples. C'est le plaisir de faire à manger (Morgane, française, 21 ans).

Il s'agit d'une recherche de santé physique, mais aussi d'une façon de penser l'acte culinaire comme un acte collectif comprenant des facteurs éthiques, économiques, 
sociaux et politiques. Ces informatrices, davantage concernées par les discours liés aux crises alimentaires, aux menaces écologiques et/ou aux évolutions de l'alimentation (comme l'industrialisation), sont soucieuses de la trajectoire du produit. Indépendamment de l'aliment et de sa composition nutritionnelle, avoir cette conscience et ce contact avec le produit crée une dynamique d'équilibre entre l'aliment, l'environnement et le mangeur, sa santé et son corps. Le fait maison serait ainsi associé à un temps alimentaire suivant un ordre perçu comme plus approprié à la santé : choisir les aliments, connaître leur provenance, savoir avec quoi ils ont été mélangés, où, comment et pour qui. Pour Lia (française, 21 ans) « bien manger » c'est :

\begin{abstract}
Prendre du plaisir avec ce que l'on mange et sentir que ça fait du bien. C'est-à-dire que j'ai mangé une bonne recette cuisinée avec amour. Je pense que quand c'est nous qui l'avons préparée, c'est forcément quelque chose qui fait du bien. On m'a appris à bien manger, à ne pas bouffer n'importe quoi, à ne pas manger entre les repas, puis à faire les choses soi-même. Comme ma mère cuisine beaucoup j'ai toujours compris que les cookies sont meilleurs, au goût et pour la santé, quand on les fait soi-même.
\end{abstract}

\title{
Activité culinaire comme partage et intégration
}

Corbeau (2002) fait appel aux notions de «socialité » et "sociabilité » afin d'expliquer le dynamisme du rapport de l'homme à la nourriture. La «socialité » représente le statut de chaque être humain vivant en société, soumis à des formes de socialisation qui le façonnent culturellement. La «sociabilité » serait le processus interactif dans lequel le mangeur choisit les manières de communication et d'échange le liant aux autres. L'activité culinaire, associée à la socialité et sociabilité, permet la communication et la création d'un lien avec autrui. Danesi (2013) définit des catégories de significations du manger: "manger pour se retrouver» et «manger pour s'intégrer ». Ces deux catégories sont opératoires pour penser l'activité culinaire, car si cuisiner permet la rencontre avec autrui, il s'agit également d'une façon de s'intégrer au sein du groupe.

L'activité culinaire comme forme de partage est davantage présente dans les discours des informatrices françaises. Au Brésil, l'acte culinaire comme acte de partage s'inscrit principalement au cours du weekend ou lors de situations festives, alors qu'en France, partager l'activité culinaire s'inscrit aussi dans le quotidien. Cette situation pourrait être liée aux contextes de commensalité de façon plus générale. Si en France la commensalité est souvent rattachée au contexte domestique, au Brésil elle est davantage associée au contexte hors foyer, n'incluant donc pas les étapes de préparation 
alimentaire. Célia (diététicienne française, 32 ans) aime «recevoir» chez elle et « cuisiner » pour ses invités :

J'aime bien préparer pour plusieurs personnes, je n'aime pas préparer le repas pour moi seule $(. .$.$) j'aime bien cuisiner et \mathrm{j}$ 'aime bien recevoir, donc $\mathrm{j}$ 'aime bien partager et faire goûter des plats aux autres, je trouve plus sympa de partager le repas.

Pour 78,4\% des français, le mot « convivialité » est associé à l'activité culinaire (Poulain, 2002b). Pfirsch (1997) a vérifié chez des français que l'idée de repas associe la notion de convivialité à la préparation domestique du repas. Fischler et Masson (2008) montrent que pour les français l'alimentation mobilise notamment le registre de la commensalité. Le repas fonctionne sur un modèle communiel, c'est-à-dire que les mangeurs partagent un repas préparé par un hôte qui « donne de lui-même ». Dans notre étude, la commensalité est revendiquée par les françaises comme une valeur apprise au sein de la famille et liée à l'identité française. Afin d'expliquer les rôles attribués à l'alimentation, Emmanuelle (diététicienne française, 26 ans) considère la convivialité en France, en l'associant à sa propre culture alimentaire :

C'est aussi convivial, puisqu'on mange avec d'autres personnes. En France c'est quelque chose de très important.

Le partage culinaire peut se faire au travers de l'échange (oral, empirique, écrit et/ou virtuel) de recettes et connaissances culinaires entre amis, colocataires, le compagnon et entre membres de la famille, principalement au travers de la mère et la grand-mère. Il s'organise au quotidien et/ou lors de rencontres familiales ou amicales, où différents individus se retrouvent pour cuisiner et/ou manger ensemble. En outre, la cuisine comme forme de partage s'inscrit tant dans l'acte de cuisiner avec que pour autrui :

Quand je cuisine, je n'aime pas cuisiner que pour moi, je préfère cuisiner pour quelqu'un (Chloé, française, 24 ans)

J'adore cuisiner, mon copain apprécie beaucoup ce que je fais, du coup c'est un plaisir partagé, faire plaisir à l'autre (Laura, française, 18 ans).

Chez les jeunes femmes, principalement, la vie en colocation favorise ces moments de partage. Si, généralement, les dynamiques de partage se mettent en place de par une affinité entre les membres, ces moments permettent, à leur tour, de renforcer ces liens. Dans certains cas, les membres de la colocation n'établissent pas de compromis dans l'organisation de l'activité culinaire et une logique de don se met ainsi en place. Dans d'autres cas, un tour de rôle s'organise suivant une logique de distribution des tâches ménagères, définie selon le temps nécessaire à leur réalisation et l'affinité de chacun vis-à-vis de l'activité : 
Je suis en colocation avec une amie, la plupart du temps je mange avec elle. C'est moi qui cuisine, parce que c'est comme ça qu'on s'est organisé, parce que j'aime bien cuisiner, elle déteste ça, elle fait le ménage (Coline, française, 18 ans).

Le partage de la cuisine est aussi la conséquence d'une division stratégique des responsabilités visant à la praticité, à l'économie de temps et d'argent. Le partage de la cuisine participe donc à l'organisation de vie en colocation.

Au sein de la famille, l'activité culinaire permet la transmission de goûts, recettes, préparations, rituels, ingrédients, histoires et habitudes familiales. Lorsque nous demandons à Anne (diététicienne française, 36 ans) si le modèle alimentaire de ses parents est une référence pour elle, elle explique :

Par rapport aux goûts et saveurs qui restent dans ma mémoire. C'est vrai qu'il y a des plats que faisait mon père, ce sont des choses que j'aime encore aujourd'hui, que j'aime faire aussi, parce que je retrouve ce goût qui me rappelle mon enfance. Après tout ce qui est autour du repas, la convivialité, le fait de se retrouver en famille, de manger ensemble, de partager le repas, c'est plus ces valeurs-là qui restent aujourd'hui.

Certaines formes d'organisation de la tâche culinaire établissent un rituel, créant un lien entre les membres de la famille. Chez les parents de Marta (brésilienne, 24 ans) ils cuisinaient ensemble tous les mercredis :

Quand on était petit, on cuisinait ensemble tous les mercredis. Il fallait avoir la discipline de penser à ce qu'on allait faire la semaine suivante et d'acheter les ingrédients avec ma mère puis de les préparer, donc chez moi tout le monde aime cuisiner.

L'activité culinaire est donc impliquée dans la construction de l'identité alimentaire, intégrant l'individu dans des groupes, familial ou de pairs. Elle participe aussi à la construction de l'identité sociale régionale ou nationale, s'inscrivant ainsi dans l'ordre de la socialité. Par exemple, les informatrices associent leur façon de cuisiner à des mots, tels que «traditionnel» ou «typique », ou à certains « marqueurs identitaires », c'est-à-dire des aliments revendiqués comme des éléments importants et/ou de base de leur modèle alimentaire. D'autres informatrices associent plus spécifiquement leur façon de cuisiner ou leur rapport au culinaire à leur pays ou région. Linda (française, 24 ans), aussi marocaine, évoque le « tajine » :

Depuis que je suis venue ici (Toulouse), j'ai appris à cuisiner, mais j'ai gardé quand même des manières de cuisiner de chez moi donc je ne fais pas mal de tajine.

Rafaela (brésilienne, 27 ans), originaire de Bahia, région réputée pour sa culture gastronomique, souligne :

Je viens d'une région où il y a beaucoup de nourritures typiques. Je pense qu'il est important de savoir cuisiner et préparer sa nourriture car je crois que cela fait partie des options culturelles, le choix de certains aliments, ces aliments portent en eux l'endroit d'où tu viens. (...) Cela est ancré dans la culture et ce que tu fais de ces aliments aussi. 
$\underline{\text { Activité culinaire comme source de plaisir }}$

L'activité culinaire est également considérée comme une manière d'éprouver $d u$ plaisir. Bien que ce plaisir puisse se ressentir durant la semaine, il est davantage associé au weekend ou aux moments festifs, lorsque le temps ne s'organise pas autour d'autres responsabilités et contraintes quotidiennes. Dans ce sens, comme le remarque Adamiec (2016), le weekend provoque un allongement du temps des préparations, qui se détache du rythme de la semaine, réinscrivant l'activité culinaire dans le temps du loisir et du partage.

Le plaisir de l'activité culinaire n'est pas tout à fait lié à l'acte de manger, mais plutôt à l'ensemble du processus de préparation de l'achat à la transformation des aliments. Il s'agit du plaisir d'être en contact direct avec la nourriture et du plaisir de pouvoir « jouer» avec les aliments, d'expérimenter de nouvelles saveurs et recettes. Il va jusqu'à exister un plaisir gustatif qui semble indissociable de l'expérience culinaire. Le plaisir de cuisiner est souvent inscrit dans une logique de convivialité. Il s'agit même d'une satisfaction symbolique, faire du bien à soi-même et aux autres, par le partage d'un bien (une préparation) conçu avec soin et qui porte en lui des valeurs reconnues par les membres du repas. Selon Emmanuelle (diététicienne française, 26 ans) l'alimentation est une source de plaisir, car :

Je prends beaucoup de plaisir à cuisiner, à manger et à le faire partager. C'est vraiment essentiel. Je reçois souvent des amis et ça ne va pas être un paquet de chips à l'apéritif, je vais toujours cuisiner.

L'activité culinaire est aussi perçue comme un plaisir car il s'agit d'un moment de détente au sein d'une routine stressante :

Ça fait un mois et demi que j'ai commencé à cuisiner. Je prépare ma nourriture le soir quand j'arrive chez moi. J'aime, pour moi c'est une façon de me déconnecter (Julia, brésilienne, 22 ans).

L'alimentation me rend bien, parce que j'éprouve du plaisir et que c'est agréable, parce que souvent ça me détend de cuisiner et de prendre du plaisir à manger ce que j'ai préparé (Célia, diététicienne française, 32 ans).

Les informatrices françaises sont plus nombreuses que les brésiliennes à concevoir l'activité culinaire comme un plaisir. Elles mettent systématiquement en avant sa dimension hédoniste et revendiquent le «goût de la cuisine ». D'autres études observent cette appréciation positive : pour 90\% des français cuisiner est un acte convivial et pour $81,6 \%$ un plaisir (Delamaire et al., 2008). Cette connexion entre plaisir et activité culinaire est au cœur du rapport des françaises à l'alimentation. Une partie des informatrices françaises valorise tellement l'activité culinaire qu'elles vont jusqu'à 
associer leur plaisir de manger à cette activité. La réponse de Nathalie (diététicienne française, 22 ans) à la question « est-ce que l'alimentation est un plaisir ? » l'illustre :

Cuisiner c'est un plaisir et manger c'est un plaisir aussi. Il n'y a aucune contrainte, franchement, c'est une passion.

Pour plusieurs françaises, cuisiner est donc une affaire de «passion» et de «bonheur» qui s'éloigne d'un vécu contraignant, davantage exprimé par les brésiliennes. En effet, nous observons que, de façon plus générale, plus que chez les brésiliennes, chez les françaises interviewées l'association entre dimension hédoniste et acte alimentaire est davantage imbriquée dans le rapport à l'alimentation. Par exemple, les informatrices françaises accordent davantage d'attention au plaisir de la table lorsqu'elles attribuent des rôles à l'acte alimentaire, et pour la majorité d'entre elles le plaisir est aussi fondamental que le rôle physiologique.

\section{Conclusion}

Cet article analyse les pratiques et les significations de l'activité culinaire chez des femmes françaises et brésiliennes. Dans un premier temps nous avons observé qu'il existe des dynamiques genrées autour de cette activité. En effet, dans les deux pays l'activité culinaire demeure davantage à la charge des femmes et la transmission des savoirs culinaires s'opère au travers de l'univers féminin. Etant donné que l'étude s'est limitée à l'analyse de discours de femmes, des travaux comparatifs portant sur des discours masculins pourraient s'avérer pertinents afin d'approfondir la compréhension de leur rapport à l'activité culinaire. Par ailleurs, puisque des changements importants sont observés dans les rôles sociaux des hommes et des femmes, des études diachroniques pourraient chercher à comprendre les transformations, au cours du temps, de leur rapport à l'activité culinaire, ainsi que les nouvelles dynamiques sociales qui se mettent en place.

Nos analyses permettent aussi de vérifier que pour les deux nationalités l'activité culinaire n'est pas seulement liée à un besoin vital nutritionnel, mais c'est aussi un acte qui porte des significations associées à la construction de l'identité socioculturelle, à l'échange avec autrui, à une forme de convivialité et de plaisir. Des différences sont pourtant observées entre les deux nationalités. L'activité culinaire occupe une place plus importante dans la vie quotidienne des informatrices françaises et ces dernières sont plus nombreuses à déclarer savoir cuisiner et aimer cette activité. Tandis que chez les françaises l'acte alimentaire est davantage signifié et expérimenté au travers de l'ensemble des étapes de préparation avec une forte valorisation du plaisir et de la 
convivialité, chez les brésiliennes l'activité culinaire est davantage perçue comme une contrainte et est moins connectée au rapport à l'alimentation. Par ailleurs, les informatrices brésiliennes accordent plus d'importance aux propriétés nutritionnelles. Du fait que l'étude s'est restreinte à des diététiciennes et des jeunes femmes étudiantes à l'université, ainsi qu'à des zones urbaines spécifiques, une approche considérant d'autres groupes sociaux, d'autres villes et/ou des zones rurales pourrait saisir la complexité des contextes nationaux, qui n'a pas été abordée dans la présente étude.

Un des apports principaux de cette étude réside dans l'approche comparative utilisée. Les comparaisons internationales s'avèrent d'intérêt pour la compréhension des modèles alimentaires, au regard d'une contemporanéité caractérisée par des changements à l'origine d'une articulation entre le global et le local. Notre comparaison révèle l'existence de grilles distinctes afin d'expérimenter, d'organiser et de signifier l'activité culinaire, s'inscrivant dans des contextes marqués par différents processus historiques et socioculturels, qui sont observables, par exemple, à travers l'âge d'émancipation ou le rapport au travail domestique. Ces contextes constituent des systèmes normatifs socioculturels inhérents à chaque modèle alimentaire. Dans ce sens, si nous pouvons identifier des éléments communs entre les deux nationalités, nous ne sommes pas face à une situation où les transformations sociétales (telles que l'industrialisation, la globalisation ou la médicalisation) fonctionnent comme des niveleuses socioculturelles.

Par ailleurs, il convient de signaler l'importance de cette étude lors du choix des contextes. En effet, nous avons constaté une asymétrie par rapport aux données existantes concernant les normes, représentations et pratiques alimentaires des deux pays, allant jusqu'à nous renseigner sur leur propre rapport à l'alimentation. Si en France, depuis les quatre dernières décennies, de nombreuses recherches ont analysé les différentes dimensions du modèle alimentaire, tant à partir d'approches quantitatives que qualitatives, au Brésil, ces études ont principalement commencé à émerger au cours des quinze dernières années. Notre travail permet ainsi de contribuer in fine à l'analyse du modèle alimentaire dans le contexte brésilien. 


\section{RÉFÉRENCES}

ADAMIEC, Camille. Devenir sain : des morales alimentaires aux écologies de soi. Tours/Rennes : PUFR/PUR, 2016.

ANIA, Association Nationale des Industries Alimentaires. Les français et l'alimentation, 2015. Disponible sur : https://www.ania.net/alimentationsante/barometre-les-francais-et-lalimentation. (Consulté le 22/08/2016).

AZEVEDO, Elaine. Food Activism. The Locavorism Perspective. Ambiente \& Sociedade, v. XVIII, n. 3, p. 80-98, 2015.

BARBOSA, Livia. Feijão com arroz e arroz com feijão: o Brasil no prato dos brasileiros. Horizontes Antropológicos, v. 13, n. 28, p. 87-116, 2007.

BARBOSA, Livia. Food and sociability on the contemporary Brazilian plate. Etnográfica, v. 14, n. 3, p. 567-587, 2010.

BLANCHET, Alain, GOTMAN Anne. L'entretien. Paris : Armand Colin, 2005.

BOAS, Marie-Hélène S.V. Ecrire la comparaison lorsque les données sont asymétriques. Une analyse de l'engagement dans les dispositifs participatifs brésiliens. Revue Internationale de Politique Comparée, v. 19, n. 1, p. 61-74, 2011.

BRITES, Jurema. Afeto e desigualdade: gênero, geração e classe entre empregadas domésticas e seus empregadores. Cadernos Pagu, v. 29, p. 91-109, 2007.

CANCLINI, Nestor G. Cultura y comunicación: entre lo global y lo local. La Plata: Universitaria de la Plata, 1997.

CARVAlHO, Luiz G.A., BASTOS, Sênia R., GIMENES-MINASSE, Maria Henriqueta S.G. Comensalidade na Família Nuclear Paulistana: 1950 a 2000. Revista Rosa dos Ventos, v. 9, n. I, p. 18-31, 2017.

CASTRO, Biancca S., YOUNG, Carlos E.F., LIMA, Guilherme R. A percepção pública de risco alimentar e os organismos geneticamente modificados no Brasil. Estudos Sociedade e Agricultura, v. 22, n. 1, p. 164-192, 2014.

COMMISSION EUROPEENNE. Que signifie être jeune dans l'Union européenne aujourd'hui ? Faits et chiffres sur les jeunes et les enfants dans l'UE. Service de presse d'Eurostat, 2015.

COLLAÇO, Janine H.L. Um Olhar Antropológico sobre o Hábito de Comer Fora. Campos, v. 4, p. 171-194, 2003.

COLLAÇO, Janine H.L. Cozinha doméstica e cozinha profissional: do discurso às 
práticas. Caderno Espaço Feminino, v. 19, n. 1, p. 143-171, 2008.

CONTRERAS, Jesus, GRACIA-ARNÁIZ, Mabel. Alimentación y cultura: perspectivas antropológicas. Barcelona : Ariel, 2005.

CORBEAU, Jean-Pierre. Itinéraires des mangeurs. Dans : CORBEAU, Jean-Pierre, POULAIN, Jean-Pierre. Penser l'alimentation : entre imaginaire et rationalité, p. 25135. Toulouse : Éditions Privat, 2002.

DANESI, Giada. Manger ensemble : les formes et les significations de la commensalité chez des jeunes adultes français, allemands et espagnols d'aujourd'hui. Thèse de Doctorat de Sociologie, Paris : EHESS, 2013.

DANIELS, Sarah, GLORIEUX, Ignasi, MINNEN, Joeri, TIENOVEN, Theun P. More than preparing a meal? Concerning the meanings of home cooking. Appetite, v. 58, n. 3 , p. 1050-1056, 2012.

DELAMAIRE, Claire, BOSSARD Corinne, JULIA, Chantal. Perceptions, connaissances et attitudes en matière d'alimentation. Dans: ESCALON, Helène, BOSSARD, Claire, BECK, François (org.) Baromètre santé nutrition 2008, p. 78-115. Paris : INPES, 2008.

DEVAULT, Marjorie L. Feeding the family. The social organization of caring as gendered work. Chicago : The University of Chicago Press, 1991.

DIAZ-MENDEZ, Cecilia, GARCIA-ESPEJO, Isabel. Eating practice models in Spain and the United Kingdom: A comparative time-use analysis. International Journal of Comparative Sociology, v. 55, n. 1, p. 24-44, 2014.

DROUARD, Alain. Les français et la table : alimentation, cuisine, gastronomie du Moyen Âge à nos jours. Paris : Ellipses, 2005.

FISCHLER, Claude. Gastro-nomie et gastro-anomie. Communications, n. 31, p. 59-61, 1979.

FISCHLER Claude. L'Homnivore. Paris : Odile Jacob, 1990.

FISCHLER Claude, MASSON Estelle. Manger : français, européens et américains face à l'alimentation. Paris : Odile Jacob, 2008.

FOURNIER, Tristan, JARTY, Julie, LAPEYRE, Nathalie, TOURAILLE, Priscille. L'alimentation : arme du genre? Journal des Anthropologues, v. 140-141, p. 19-49, 2015 .

GALINDO, Flavia, PORTILHO, Fatima. "O peixe morre pela boca": como os consumidores entendem os riscos dos agrotóxicos e dos transgênicos na alimentação. 
Sustentabilidade em Debate, v. 6, n. 2, p. 73-87, 2015.

GARABUAU-MOUSSAOUI, Isabelle. La cuisine des jeunes : désordre alimentaire, identité générationnelle et ordre social. Anthropology of food, [Online], Issue 0, 2001.

GATLEY, Andy, CARAHER, Martin, LANG, Tim. A qualitative, cross cultural examination of attitudes and behaviour in relation to cooking habits in France and Britain. Appetite, v. 75, p. 71-81, 2014.

GIDDENS, Anthony. Modernity and Self-Identity: Self and Society in the Late Modern Age. Standford : Stanford University Press, 1991.

GOMES, Paula. Emprego doméstico e a arquitetura da desigualdade. Ciência e Cultura, v. 68, n. $2,2016$.

HERPIN, Nicolas. Le repas comme institution : compte rendu d'une enquête exploratoire. Revue Française de Sociologie, v. 29, n. 3, p. 503 521, 1988.

IBGE, Instituto Brasileiro de Geografia e Estatística. Coordenação de Trabalho e Rendimento. Pesquisa de Orçamentos Familiares (POF). Rio de Janeiro, p. 150, 2011.

JOMORI, Manuela M., VASCONCELOS, Francisco A.G., BERNARDO, Greyce L., UGGIONI, Paula L., PROENÇA, Rossana P.C. The concept of cooking skills: A review with contributions to the scientific debate. Revista de Nutrição, v. 31, n. 1, p. 119-135, 2018.

JOURDAN, Christine, RILEY Kathleen, C. Présentation : la glocalisation alimentaire. Anthropologie et Sociétés, v. 37, n. 2, p. 9-25, 2013.

KAUFMANN, Jean-Claude. Casseroles, amour et crises. Ce que cuisiner veut dire. Paris : Armand Colin, 2005.

LEVI-STRAUSS, Claude, Mythologiques, $t$. I : Le Cru et le cuit. Paris : Plon, 1964.

LOISEL, Jean-Pierre, COUVREUR, Agathe. De la transmission à l'apprentissage des savoir-faire culinaires: regards croisés de filles et de mères. Crédoc, Cahier de Recherche, n. 198, 2004.

MACIEL, Maria E. Uma cozinha à brasileira. Estudos Hitóricos, n. 33, p. 25-39, 2004.

MALYSSE, Stephen. Em busca dos (H)alteres-ego: olhares franceses nos bastidores da corpolatria carioca. Dans : GOLDENBERG, Miriam (org.) $\mathrm{Nu}$ e Vestido. Rio de Janeiro : Record, p. 79-138, 2002.

MARIE, Patricia. Hommes et femmes dans l'apprentissage et la transmission de "l'art culinaire ». Paris : L'Harmattan, 2014.

MASSON, Estelle. Vers une alimentation sur mesure ? Régimes personnels et habitudes 
collectives. Dans: FISCHLER, Claude (org.) Les alimentations particulières. Mangerons-nous encore ensemble demain? p. 236-245. Paris : Odile Jacob, 2013. MATHE, Thierry, TAVOULARIS, Gabriel, PILORIN, Thomas. La gastronomie s'inscrit dans la continuité du modèle alimentaire français. Crédoc, Cahier de Recherche, n. 267, 2009.

MATHÉ, Thierry., BELDAME, Diane, HÉBEL, Pascale. Évolution des représentations sociales du bien manger. Crédoc, Cahier de Recherche, n. 316, 2014.

MAUSS, Marcel. Les techniques du corps. Dans : MAUSS, Marcel. Sociologie et Anthropologie. Paris : PUF, 2009 [1934].

MEAD, Margareth. Contextos culturales de las pautas de nutrición. Dans : MEAD, Margareth. (org.) La antropología y el mundo contemporáneo. Buenos Aires: Siglo Veinte, 1971.

MENNELL, Stephen, MURCOTT, Anne, OTTERLOO, Anneke H. The sociology of food: eating, diet and culture. London : Sage publications, 1993.

MORIN, Edgar. Le paradigme perdu : la nature humaine. Paris : Le Seuil, 1973.

PETONNET, Colette. L'observation flottante. L'exemple d'un cimitière parisien. Études d'anthropologie urbaine, n. 4, p. 37-47, 1982.

PETTINGER, Claire, HOLDSWORTH, Michelle, GERBER, Mariette. Meal patterns and cooking practices in Southern France and Central England. Public Health Nutrition, v. 9, n. 8, p. 1020-1026, 2006.

PFIRSCH Jean-Vincent. La saveur des sociétés : sociologie des goûts alimentaires en France et en Allemagne. Rennes : Press Universitaires de Rennes, 1997.

POULAIN Jean-Pierre. Sociologies de l'Alimentation. Paris : PUF, 2002a.

POULAIN Jean-Pierre. Manger Aujourd'hui : attitudes, normes et pratiques. Toulouse : Privat, 2002b.

ROBERTSON, Roland. Globalization: Social Theory and Global Culture. London: Sage, 1992.

ROZIN, Paul, FISCHLER, Claude, IMADA, Sumio, SARUBIN, Allison, WRZESNIEWSKI, Amy. Attitudes to food and the role of food in life in the USA, Japan, Flemish Belgium and France: Possible implications for the diet-health debate. Appetite, v. 33, n. 2, p. 163-180, 1999. 
SANT'ANNA, Denise B. Brésil : le beau, le mince, le sain. Dans : FISCHLER, Claude, MASSON, Estelle (org.) Manger : français, européens et américains face à l'alimentation. Paris : Odile Jacob, p. 305-313, 2008.

SANTOS, Ligia A.S.S. O corpo, o comer e a comida: un estudo sobre as práticas corporais alimentares a partir da cidade de Salvador - Bahia. Salvador : EDUFBA, 2008 .

SHIELDS-ARGELÈS, Christy. Du discours au récit. Approche comparative du rapport entre alimentation et constructions identitaires en France et aux États-Unis. Thèse de Doctorat de Sociologie, EHESS, France, 2012.

SCHUBERT, Maicon, SCHNEIDER, Sergio, DÍAZ-MÉNDEZ, Cecilia. O “comer fora de casa" no Brasil, Reino Unido e na Espanha: uma revisão das bases de dados estatísticos oficiais e perspectivas para comparação. Estudos Sociedade e Agricultura, v. 25, n. 2, p. 277-304, 2017.

SHORT, Frances Domestic cooking skills. What are they? Journal of the HEIC, v. 10, n. 3, p. 13-22, 2003.

SICART Daniel. Les professionnels de la santé au lère Janvier 2014. Document de travail, série statistiques, 189, 2014.

TNS Sofres. Comprendre les attentes des consommateurs en matière d'alimentation. Food 360. Paris, 2014.

VERDALLE, Laure, VIGOUR, Cécile, LE BIANIC, Thomas. S'inscrire dans une démarche comparative : enjeux et controverses. Terrains \& Travaux, n. 21, p. 5-21, 2012.

VIGOUR, Cécile. La comparaison dans les sciences sociales. Paris : La Découverte, 2005.

WARDE, Alan. Convenience food: space and timing. British Food Journal, v. 101, n. 7, p. 518-527, 1999.

WARDE, Alan, CHENG, Shu-Li, OLSEN, Wendy, SOUTHERTON, Dale. Changes in the practice of eating. A comparative analysis of time-use. Acta Sociologica, v. 50, n. 4 , p. 363-385, 2007. 
Remerciements : les auteurs remercient la Prof. Janine Collaço pour les lectures attentives de l'article, ainsi que le Prof. Jean-Pierre Poulain et le Prof. Jesús Contreras pour les orientations données au cours du développement du travail de recherche. Cette recherche a reçu le soutien de la Fondation Nestlé France et de l'Eurorégion PyrénéesMéditerranée.

Recebido 21/08/2019

Aprovado 11/12/2019 